\title{
A visão de estudantes de medicina e enfermagem sobre a integralidade na Atenção Primária à Saúde
}

\author{
Comprehensiveness in the Primary Health Care System: \\ understanding of medical and nursing students
}

\section{Thiago Luccas Correa dos Santos Gomes', Elza de Fátima Ribeiro Higa ${ }^{2}$, Antônio Henrique Rodrigues Passos ${ }^{3}$, Márcia Oliveira Mayo Soares ${ }^{4}$, Márcia Aparecida Padovan Otani ${ }^{5}$, Bernardino Geraldo Alves Souto ${ }^{6}$}

\begin{abstract}
'Mestre em Ensino em Saúde. Assistente de ensino na Faculdade de Medicina de Marília. Marília, São Paulo, Brasil. †lcsgomes@gmail.com ${ }^{2}$ Doutora em Enfermagem Fundamental. Docente na Faculdade de Medicina de Marília. Marília, São Paulo, Brasil. hirifael@gmail.com ${ }^{3}$ Doutor em Medicina e Ciências da Saúde. Docente na Faculdade de Medicina de Marília. Marília, São Paulo, Brasil. ahrpassos@gmail.com ${ }^{4}$ Mestre em Ensino em Saúde. Assistente de ensino na Faculdade de Medicina de Marília. Marília, São Paulo, Brasil. mmayosoares@gmail.com ${ }^{5}$ Doutora em Saúde Coletiva. Docente na Faculdade de Medicina de Marília. Marília, São Paulo, Brasil. mm-otani@famema.br ${ }^{6}$ Doutor em Medicina/Ciências da Saúde. Professor associado do Departamento de Medicina na Universidade Federal de São Carlos. São Carlos, São Paulo, Brasil. bernardino@viareal.com.br
\end{abstract}

\begin{abstract}
Resumo I O objetivo desta pesquisa foi compreender a integralidade pela ótica de estudantes de medicina e enfermagem inseridos na Atenção Primária à Saúde. Ela tem como pressuposto norteador que há necessidade de compreensão do cuidado nessa perspectiva pois apresenta impacto e relevância na formação de futuros profissionais de saúde. Pesquisa do tipo exploratória e descritiva, com abordagem qualitativa. Foram entrevistados oito estudantes e os dados foram interpretados pela Análise de Conteúdo na Modalidade Temática. Emergiram três categorias temáticas: "A articulação entre teoria e prática favorece o ensino-aprendizagem sobre a integralidade", "Cuidado centrado na pessoa e seu contexto familiar e comunitário permite o desenvolvimento da integralidade" e "Falta de estrutura da UPP e das Unidades de Saúde deixam lacunas na aprendizagem da integralidade". Os resultados indicam que os estudantes vislumbram o ensino-aprendizagem tendo em vista a compreensão do cuidado na perspectiva da integralidade.
\end{abstract}

Palavras-chave: Atenção primária à saúde; Integralidade em saúde; Educação em saúde; Gestão em saúde; Assistência integral à saúde.

\begin{abstract}
This research analyzed the understanding of the concept of "comprehensiveness" from the medicine/ nursing students' perspective integrated in the Primary Health Care System (PHC). The guiding assumption is the need to understand the comprehensiveness in PHC due to its impact and relevance in future health professionals training. This is an exploratory, descriptive study, with a qualitative approach. Eight students were interviewed and the data was interpreted using the Content Analysis method in the Thematic Modality. The result was the emergence of three thematic categories: "The interrelationship between theory and practice promotes the teaching-learning process about comprehensiveness"; "Both people-centered care and the community/family allude to the development of comprehensiveness and, "Inadequacy in UPP and Healthcare facilities structure hinders the learning of comprehensiveness. The findings suggest the students grasp the teaching-learning process in terms of the understanding of care from the comprehensiveness perspective.
\end{abstract}

Keywords: Primary health care; Comprehensive health care; Health education; Health management. 


\section{Introdução}

As transformações sociais, econômicas, políticas e tecnológicas ocorridas ao longo do século $X X$ e início do século XXI trazem mudanças significativas para a formação de profissionais de saúde em âmbito mundial (Gomes, Higa, Passos, Otani, Soares, \& Souto, 2017).

O Brasil promulga a constituição federativa no ano de 1988 que garante a saúde como direito universal e considera a integralidade como um dos princípios do Sistema Único de Saúde (SUS) (Brasil, 1990; Paim, Travassos, Almeida, Bahia, \& Macinko, 2011 ).

A formação em saúde que ocorria centrada no hospital e nas especialidades começa a se ajustar a à luz das Diretrizes Curriculares Nacionais (DCN) no ano de 2001 e incorpora a necessidade de um perfil profissional generalista, humanista, crítico e reflexivo com a prática profissional voltada às principais necessidades de saúde da população, sob a perspectiva da integralidade da assistência (Gomes et al., 2017). No contexto da educação médica existem diversos movimentos voltados à construção de novos perfis de competência profissional (Hafner et al., 2010).

Uma faculdade do interior do Estado de São Paulo desenvolve a formação profissional em consonância com as diretrizes do SUS e com as DCN utilizando um currículo que tem como eixo direcionador o desenvolvimento de competência profissional de acordo com os princípios de metodologias ativas de aprendizagem (Gomes et al., 2017; Faculdade de Medicina de Marília, 2016). As duas primeiras séries dos cursos de medicina e enfermagem, que na Famema, nas atividades da prática profissional caminham juntas, buscam desenvolver habilidades nas áreas do cuidado individual, do cuidado coletivo, de gestão dos serviços de saúde e de iniciação científica (Gomes et al., 2017; Faculdade de Medicina de Marília, 2016). Para alcançarem os desempenhos propostos devem realizar uma série de tarefas articulando os aspectos psicomotores, afetivos e cognitivos em cenários diversos da rede de cuidados à saúde. Estão fundamentados na lógica da vigilância em saúde, com ênfase na Atenção Primária à Saúde (APS)
A estrutura das séries é composta pelas Unidades de prática profissional (UPP), Unidade Educacional Sistematizada (UES) e Unidade Educacional Eletiva (UEE). A UPP conta com o Laboratório de Prática Profissional (LPP) onde são realizadas atividades de simulação da prática previamente organizadas pelos docentes da série. A UPP estimula a problematização das situações desafiadoras enfrentadas na vida real e na simulação enquanto as tutorias inseridas na UES são realizadas em pequenos grupos de estudantes utilizando-se aprendizagem baseada em problemas (Gomes et al., 2017).

Diante do trabalho docente realizado com estudantes no cenário de prática e das atuais políticas nas áreas de saúde e educação, essa pesquisa tem como objeto a compreensão da Integralidade do cuidado em saúde na perspectiva dos estudantes dos Cursos de Enfermagem e Medicina, de uma faculdade pública, inseridos na APS. Surgiu da seguinte inquietação: como os estudantes compreendem a integralidade dentro da APS?

A compreensão da integralidade na APS pode contribuir com a melhoria da qualidade do cuidado, respeitando as necessidades de saúde da população. Dessa maneira, equipes de saúde e gestores devem desenvolver suas ações possibilitando o cuidado centrado na pessoa e sua liderança capaz de revolucionar o processo de transformação do modelo assistencial vigente no país (Gomes et al., 2017 ; Paim et al., 2011).

O Ministério da Saúde desde 1995 em parceria com gestores estaduais e municipais com objetivo estratégico de reorientar o modelo e universalizar a APS para ações de promoção, prevenção e assistência à saúde, de maneira integrada, em um território definido, estimula a opção pelo Programa de Saúde da Família (PSF). A partir de 1999, o PSF passou a ser considerado como uma estratégia estruturante dos sistemas municipais de saúde - a Estratégia de Saúde da Família (ESF) com vistas a imprimir uma nova dinâmica na organização dos serviços e ações de saúde (Gomes, 2015; Paim et al., 2011).

A ESF é o programa de qualificação da APS de maior sucesso da história do Brasil. A ESF é a estratégia para reorganização do modelo assistencial do SUS, o que vai ao encontro do relatório da Or- 
ganização Mundial da Saúde de 2008, que destacou o Brasil como exemplo, citando as iniciativas da ESF e dos Observatórios de Recursos Humanos em Saúde como modelos a serem seguidos (Gomes, 2015; Paim et al., 2011; Organização Pan Americana da Saúde, 2009).

A Política Nacional de Atenção Básica (PNAB) brasileira, publicada pela primeira vez em 2006, revisada em 2012 e em 2017, define a APS como "um conjunto de ações de saúde, no âmbito individual e coletivo, que abrange promoção e proteção da saúde, prevenção de agravos, diagnóstico, tratamento, reabilitação e manutenção da saúde. com o objetivo de desenvolver uma atenção integral que impacte na situação de saúde e autonomia das pessoas e nos determinantes e condicionantes de saúde das coletividades. É desenvolvida por meio do exercício de práticas de cuidado e gestão, democráticas e participativas, sob forma de trabalho em equipe, dirigidas a populações de territórios definidos, pelas quais assume a responsabilidade sanitária, considerando a dinamicidade existente no território em que vivem essas populações. Utiliza tecnologias de cuidado complexas e variadas que devem auxiliar no manejo das demandas e necessidades de saúde de maior frequência e relevância em seu território, observando critérios de risco, vulnerabilidade, resiliência e o imperativo ético de que toda demanda, necessidade de saúde ou sofrimento devem ser acoIhidos [...] É o contato preferencial dos usuários com os sistemas de saúde. Orienta-se pelos princípios da universalidade, acessibilidade e coordenação do cuidado, vínculo e continuidade, integralidade, responsabilização, humanização, equidade, e participação social. A atenção básica considera o sujeito em sua singularidade e inserção sociocultural, buscando produzir a atenção integral". ( Gomes, Higa, Passos, Otani, \& Souto, 2016).

Na literatura internacional, Barbara Starfield define a APS como "a atenção de primeiro contato, contínua, abrangente e coordenada que se proporciona à população sem distinção de gênero, ou enfermidade, ou sistema orgânico"(Starfield, 2002, p. 46). Descreve também os atributos específicos: acesso, porta de entrada, vínculo (longitudinality), elenco integral/ abrangente de serviços (comprehensiveness), coordenação (ou integração dos serviços), enfoque familiar e orientação para a comunidade (Starfield, 2002).
Pesquisas desenvolvidas em diversos países demonstram que 0 investimento em APS de qualidade é favorável a uma série de desfechos clínicos o que gera impacto positivo em indicadores de saúde e na satisfação das necessidades da população (Gomes, 2015). Macinko e Lima-Costa demonstram que os serviços de saúde brasileiros desde 1998 a 2008 estão mais justos, principalmente oferecendo acesso e assistência na atenção primária e saúde da família (Macinko, 2012).

Apesar de todos os avanços, alguns desafios estão postos para o futuro da APS no SUS, entre eles, a formação e educação permanente, a consolidação da APS como ordenadora do sistema e a comunicação com a sociedade brasileira. Vencer os desafios apontados é obrigação desta na manutenção de sua maior política de inclusão social, que é o SUS orientado pela APS. Nesse sentido, Mendes destaca que "o ciclo da atenção básica à saúde caracteriza-se pela emergência e consolidação do PSF". Avançar do PSF para a ESF convoca um novo ciclo que exige respostas complexas, profundas e sistêmicas (Mendes, 2012).

Embora a Saúde da Família seja a estratégia prioritária para expansão e consolidação da APS, a cobertura desse modelo de atenção no Brasil não é universal, pois $62,54 \%$ da população, por volta de 121.313 .472 brasileiras e brasileiros estão cadastrados na ESF. Sobram, portanto, cerca de $40 \%$ de cidadãos que ainda buscam os serviços de urgência e emergência, as unidades básicas tradicionais, os serviços privados de pagamento direto e os planos e convênios de saúde, regulados pela Agência Nacional de Saúde Suplementar (ANS) (Gomes, 2015).

Um dos grandes desafios dos sistemas universais e integrais, como o SUS, é a formação de recursos humanos (RH) para o atendimento das necessidades de saúde da população. Para isso é necessário planejamento estratégico e investimento nessa formação, tendo em vista o alcance de uma cobertura universal. $O$ número de profissionais de APS deve ser adequado, garantindo a ampliação e o fortalecimento de conhecimentos, habilidades e atitudes necessários a uma atuação competente (Organização Pan Americana da Saúde, 2009). 
A ordenação da formação de RH na área da saúde está explicitada na Constituição Federativa do Brasil e os serviços de saúde devem servir de campos para o ensino e a pesquisa, local de integração da assistência, gestão e formação em saúde. Nesse mesmo sentido, surgiu uma proposta conhecida como Quadrilátero da Formação, que colocaria além dos serviços e da assistência, algumas ferramentas de planejamento para a formação em saúde na lógica da educação em serviço e educação permanente em saúde (Gomes et al., 2017, Brasil, 1990).

A articulação entre o ensino e o serviço têm se fortalecido em diversos municípios após a legislação brasileira propor a criação de diretrizes para a celebração dos Contratos Organizativos de Ação Pública Ensino-Saúde (COAPES) para o fortalecimento da Integração Ensino, Serviços e Comunidade no âmbito do SUS (Gomes et al., 2016).

\section{Objetivos}

Compreender a integralidade pela ótica de estudantes de medicina e enfermagem inseridos na Atenção Primária à Saúde.

\section{Metodologia}

\section{Tipo de estudo}

Trata-se de uma pesquisa exploratória e descritiva, com abordagem qualitativa. $O$ métdo qualitativo é o mais adequado a este estudo, considerando que esse método é mais empregado para investigar grupos e segmentos delimitados e focalizados, de histórias sociais, sob a ótica dos atores. (Minayo, 2013).

O método qualitativo trabalha com o universo dos significados, dos motivos, das aspirações, das crenças, dos valores e das atitudes. Esse conjunto de pluralidade humana faz parte do contexto do ser social, pois o ser humano é diferente no seu modo de agir e vivenciar as experiências (Minayo, 2013,p. 21).

\section{Participantes}

Participaram oito estudantes dos cursos de Enfermagem e Medicina, sendo cinco do curso de medicina, com idade entre 21 e 28 anos; três do curso de enfermagem com idade entre 18 a 23 anos, que atuaram nas Unidades de Prática Profissional (UPP) da primeira e segunda série no ano de 2014 inseridos na APS.

A amostra foi determinada, intencionalmente e por conveniência.

\section{Instrumento para coleta de dados}

A coleta de dados foi realizada por meio de entrevista com uma pergunta aberta norteadora: qual a sua compreensão sobre a integralidade no contexto da Unidade de Prática Profissional e como ela se insere na aprendizagem?

\section{Procedimento de coleta dos dados}

As entrevistas foram realizadas na Faculdade de Medicina de Marília, em datas e horários previamente agendados com os estudantes. Tiveram duração média em torno de dez minutos, foram gravadas em áudio e transcritas pelo próprio pesquisador. Os participantes concordaram com o Termo de Consentimento Livre Esclarecido (TCLE), considerando o que se preconiza na Resolução 466/2012, do Conselho Nacional de Saúde (Brasil, 2013). Aprovada pelo Comitê de Ética em Pesquisa Envolvendo Seres Humanos da Faculdade de Medicina de Marília, sob o número de protocolo 513.764 .

\section{Procedimento de análise dos dados}

Os dados obtidos foram analisados pela Técnica de Análise de Conteúdo na Modalidade Temática (Bardin, 2012), organizada em três etapas: pré-análise, categorização e interpretação dos dados. O tema permite multiplicidade de relações, podendo ser concebido por um resumo, uma frase, ou uma palavra. A análise temática visa encontrar os núcleos de sentido de uma comunicação (Bardin, 2012). 


\section{Resultados e Discussão}

Da análise dos dados obtidos por meio das entrevistas emergiram três categorias temáticas.

A primeira categoria "A articulação entre teoria e prática favorece $\circ$ ensino-aprendizagem sobre a integralidade" surge a partir da visão dos estudantes a respeito da importância de integração entre teoria e prática nos cenários reais de ensino-aprendizagem. Por meio do contato precoce com as famílias, unidades e equipes de saúde, os estudantes conhecem o Sistema Único de Saúde, os princípios e a organização do processo de trabalho, interagem com os problemas mais comuns que afligem a maioria da população e percebem a imprevisibilidade e complexidade das situações que podem surgir no nível de atenção primária.

"Ontem na tutoria, a gente estava estudando sobre humanização, que tem tudo a ver. A integralidade é um dos pressupostos do SUS. Quando a gente estava estudando humanização, falou sobre esse cuidado integral da pessoa. Hoje, na LPP, a gente teve dificuldade porque a gente não olhou o paciente com esse olhar de integralidade, de olhar pro aspecto físico, de perceber os traços dele." (E4)

Esse caráter de complementaridade das unidades educacionais, tanto nas tutorias quanto no LPP (ambientes protegidos e teóricos em sua essência), trazem um contraste com a realidade da vivência na UPP. Nesse sentido, percebe-se a validade da diversificação e a importância do contexto de cada vivência por parte dos estudantes, do que se depreende o caráter da aprendizagem significativa (Moreira, 2011).

"o SUS tem a equipe do NASF, na unidade, tem a enfermeira, o dentista, tem toda uma equipe cujo objetivo é a promoção da saúde". (E3)

Neste trecho, por exemplo, percebe-se que a promoção de saúde é o objetivo comum tanto à equipe de referência quanto à equipe matricial na APS. Nos desempenhos da área de gestão e organização do processo de trabalho em saúde o destaque é para a importância do planejamento do processo de trabalho junto à equipe, aproximando os es- tudantes dos princípios do SUS e da ESF (Gomes, 2015; Moreira, 2011).

Na segunda categoria temática "Cuidado centrado na pessoa e seu contexto familiar e comunitário permite o desenvolvimento da integralidade", os estudantes se manifestaram em relação aos saberes necessários para o desenvolvimento da integralidade e revelaram que a compreensão sobre um dos prismas da integralidade está baseada na prática do vínculo, no uso de tecnologias relacionais ou leves, na compreensão da pessoa e da família como um todo, inseridas em seu contexto de vida.

"Tecnologia leve e o contexto da saúde primária seria o contexto de baixa tecnologia dura, ou seja,

baixa aquisição de equipamentos, p. ex. raio $x$, tomografia e ressonância magnética, mas o contexto de compreensão da família, prevenção e promoção de saúde." (E 1)

"Em relação às famílias a gente tem um vínculo muito forte, aprende muito, criou um vínculo com as famílias que não quer largar. Já pegou um compromisso do cuidar por causa da nossa facilitadora.." (E3)

Sem vínculo e responsabilização com as famílias e a comunidade, a APS sofre sérios riscos de não alcançar seus objetivos nos sistemas de saúde. Esses pressupostos da ação em saúde também aparecem com destaque na PNAB (Gomes et al., 2017), pois além de abranger a adscrição de clientela, o cuidado longitudinal oferece a oportunidade de estabelecimento de relações de confiança e contínuas ao longo do tempo, sempre mediadas por subjetividades (Ayres, 2011; Cecilio, 2011). Pelo menos 60\% das consultas médicas estão relacionadas aos eventos estressantes da vida, o que significa que quando uma pessoa procura um serviço está sinalizando um pedido de ajuda (Gomes, 2015).

"É um conjunto de ações que você tem que olhar a pessoa como um todo, não só como uma doença, é esse conjunto mesmo que busca olhar a pessoa como um todo [...] inserir a pessoa no contexto.." (E4)

conceito de saúde no modelo da Vigilância à Saúde aproxima as práticas da integralidade para - desenvolvimento das competências necessárias no cenário de APS, especificamente no âmbito da 
clínica (Anderson \& Rodrigues, 2012), mas também podendo ser desenvolvidas desde as séries iniciais (Marin et al., 2010).

Em relação à orientação familiar, os diferentes ciclos de vida se caracterizam pelo momento de maior vulnerabilidade para o aparecimento de sintomas, o que torna esse reconhecimento como uma ferramenta potente de cuidado na APS (Anderson \& Rodrigues, 2012).

Em investigação da integralidade na APS no município de São Paulo entre gestores, profissionais e usuários, encontrou-se dificuldade de vínculo por não ter tempo e o estresse estar sempre presente no cotidiano das equipes (Gomes, 2015). Nesse sentido a fala do primeiro estudante reflete o entendimento das tecnologias de cuidado para a integralidade, opondo-se a dureza dos equipamentos à leveza dos relacionamentos, no entanto a tecnologia dura, além de mais cara, ainda exerce um fetiche nos estudantes de medicina. Permanece, portanto, o desafio aos sistemas de saúde valorizarem mais a formação humanística e o cuidado centrado na pessoa como ferramentas clínicas dos especialistas em APS (Feverwerker, 2011; Gomes, 2015).

A terceira categoria temática "Falta de estrutura da UPP e das Unidades de Saúde deixam lacunas na aprendizagem da integralidade", evidenciou que alguns estudantes indicam fragilidades encontradas nesse cenário. Considerando que este trabalho foi realizado em uma das primeiras escolas a implementar mudanças curriculares e a utilizar métodos de ensino-aprendizagem inovadores, as compreensões trazidas pelos estudantes tornam-se extremamente significativas.

"Se dependesse da USF, a gente já teria dado um "migué" de não estudar, mas como se criou uma amizade muito boa entre o grupo, o mesmo quer mostrar mais e mais estudo. É uma ideia muito legal na teoria, mas se não tiver um professor bom, qualificado, e uma USF que acolhe, fica muito deficiente. Talvez não se tornasse um processo prazeroso. Torna-se um peso. Muitos colegas criticam "sete meses na USF, eu vou lá e não entendo nada e vou embora. Eu preferia ficar em casa". (E3)
Existem diversos obstáculos existentes nos serviços de saúde quando estão mais voltados ao atendimento da população do que aos processos de formação e capacitação. A fala também enfatiza que os docentes funcionam como modelos de como colocar a teoria em prática, pois apenas estar na comunidade não favorece a aprendizagem se os objetivos educacionais não estiverem claros e coerentes com a proposta da UPP (Gomes et al., 2017).

"Eu acho que funciona muito bem essa integralidade, mas algumas questões ficam um pouco a desejar. Logo de início, no primeiro ano, quando a gente começa a fazer as visitas, não tem muito conhecimento de nada do que é pra fazer na casa da família, acho que fica muito aberto, que o vínculo pode ser feito de diversas formas. No primeiro contato, fica muito estranho, eu não conheço a família e eu mesmo não sei o que eu estou fazendo lá." (E7)

Embora a mudança ocorrida pelo currículo orientado para o desenvolvimento da competência profissional seja destaque no cenário nacional de reformas dos sistemas educacionais, o caminho da aprendizagem deve ser trilhado pelos maiores interessados nessa mudança - docentes e estudantes. Nesse sentido, a realidade da rotina - ações e serviços - nas Unidades de Saúde da Família é não estruturada, impondo grandes desafios ao processo de ensino-aprendizagem (Gomes et al., 2016; Gomes et al., 2017).

Na ótica dos egressos de um curso de medicina a inserção nos serviços de APS possibilitou o conhecimento da organização e do funcionamento do serviço de saúde, do contexto de vida dos usuários, além de facilitar a criação do vínculo e o desenvolvimento do raciocínio clínico. Eles destacam, no entanto, que a imaturidade do estudante nas séries iniciais impede maior aproveitamento da vivência. Essa percepção reforça e se alinha às novas diretrizes curriculares para os cursos de medicina que propõe $35 \%$ da carga horária do internato desenvolvidas na APS. (Gomes et al., 2016).

De acordo com a literatura, as limitações para maior integração entre a universidade e os serviços ocorrem por conta de processos de trabalho distintos, de um lado a universidade seria o local onde se produz conhecimento, "o saber", e de outro os serviços 
voltados para a produção da assistência, "o fazer" (Carvalho, Duarte, \& Guerrero, 2015). Essa dicotomia leva a refletir sobre o significado do "saber ser" e a necessidade de integração de todos esses aspectos na formação de docentes e estudantes e de como o professor pode se tornar qualificado para o desenvolvimento da integralidade na APS, cenário privilegiado de ensino-aprendizagem.

\section{Conflitos de interesses}

Todos os autores declaram não haver conflitos de interesses que possam influenciar ou ter influenciado na pesquisa e no relato de seus resultados.

\section{Conclusões}

Considerando o objetivo dessa pesquisa, é importante destacar o relevante papel do método qualitativo (Minayo, 2013) e da técnica de Análise de Conteúdo na modalidade temática (Bardin, 2012) que produzem sentidos e significados na diversidade de amostragem presentes no mundo acadêmico, que permitiram ampliar o olhar para compreensão da integralidade, no período inicial da graduação, em relação ao contexto da Atenção Primária à Saúde.

Os estudantes compreendem e praticam a integralidade dentro do processo ensino-aprendizagem na UPP, orientados por docentes qualificados e por unidades de saúde acolhedoras. A mudança curricular e o perfil profissional orientado por competência demandam encontros regulares de Educação Permanente no sentido de proporcionar ainda maior integração ensino-serviço-comunidade.

A compreensão da integralidade como um princípio do SUS apontados e desenvolvidos pelos participantes revela a importância da prática no cenário de APS para a formação dos futuros profissionais. Os resultados deste estudo demonstram a importância do trabalho em equipe multiprofissional e a oportunidade do reconhecimento da integralidade para a melhoria do cuidado prestado.

\section{Referências}

Anderson, M. I. P., \& Rodrigues, R. D. (2012). Integralidade na prática do médico de família na Atenção Primária à Saúde. In G. Gusso \& J. M. C. Lopes (Eds.), Tratado de medicina de família e comunidade: princípios, formação e prática (pp. 71-83). Porto Alegre: Artmed.

Ayres, J. R. C. M. (2011). Cuidado: trabalho e interação nas práticas de saúde. Rio de Janeiro: CEPESC/UERJ/IMS/ ABRASCO.

Bardin, L. (2012). Análise de conteúdo. São Paulo: Edições 70.

Lei n. 8080 , de 19 de setembro de 1990. Dispõe sobre as condições para a promoção, proteção e recuperação da saúde, a organização e o funcionamento dos serviços correspondentes e dá outras providências. Recuperado de http://www.planalto.gov.br/ccivil_03/ leis/18080.htm

Resolução n. 466, de 12 de dezembro de 2012. Aprova as diretrizes de normas regulamentadoras de pesquisas envolvendo seres humanos. Recuperado de http:// bvsms.saude.gov.br/bvs/saudelegis/cns/2013/ res0466_12_12_2012.html

Carvalho, S. B. O., Duarte, L. R., \& Guerrero, J. M. A. (2015). Parceria ensino e serviço em unidade básica de saúde como cenário de ensino-aprendizagem. Trabalho, Educação e Saúde, 13(1), 123-144. Recuperado de http://www.scielo.br/pdf/tes/v13n1/1981-7746tes-1981-7746-sip00026.pdf. doi: 10.1590/19817746-sip00026

Cecilio, L. C. O. (2011). Apontamentos teórico-conceituais sobre processos avaliativos considerando as múltiplas dimensões da gestão do cuidado em saúde. Interface (Botucatu), 15(37), 589-599. Recuperado de http:// www.scielo.br/pdf/icse/v15n37/a21v15n37.pdf. doi: 10.1590/S1414-32832011000200021

Faculdade de Medicina de Marília. (2016). Necessidades de Saúde 2 e prática profissional 2: 2a série dos Cursos de Medicina e enfermagem. Marília: Faculdade de Medicina de Marília.

Feuerwerker, L. C. M. (2011). A cadeia do cuidado em saúde. In J. J. N. Marins, \& S. Rego (Orgs). Educação médica: gestão, cuidado, avaliação, 99-1 13. São Paulo: Hucitec.

Gomes, T. L. C. S. (2015). Atenção primária à saúde e integralidade: articulação entre gestão e ensinoaprendizagem (Dissertação de mestrado). Faculdade de Medicina de Marília, SP, Brasil. 
Gomes, T. L. C. S., Higa, E. F. R., Passos, A. H. R., Otani, M. A. P., \& Souto, B. G. A. (2016). Compreensão da integralidade sob a perspectiva de docentes inseridos na atenção primária à saúde. In Atas Congresso Ibero-Americano em Investigação Qualitativa em Saúde. Porto, Portugal, 5. Recuperado de http:// proceedings.ciaiq.org/index.php/ciaiq2016/article/ view $/ 777 / 764$

Gomes, T. L. C. S., Higa, E. F. R., Passos, A. H. R. , Soares, M. O. M., Otani, M. A. P., \& Souto, B. G. A. (2017) Integralidade na Atenção Primária à Saúde: compreensão dos estudantes de medicina e enfermagem. In Atas do Congresso Ibero-Americano em Investigação Qualitativa em Educação. Salamanca, Espanha, 6. Recuperado de http://proceedings.ciaiq. org/index.php/ciaiq2017/article/view/1355

Hafner, M. L. M. B., Moraes, M. A. A., Marvulo, M. M. L., Braccialli, L. A. D., Carvalho, M. H. R., \& Gomes, R. (2010). A formação médica e a clínica ampliada: resultados de uma experiência brasileira. Ciência \& Saúde Coletiva, 15(Supl 1), 1715-1724. Recuperado de http://www.scielo.br/pdf/csc/v15s1/083.pdf. doi: 10.1590/S1413-81232010000700083

Macinko J., \& Lima-Costa M.F. (2012). Horizontal equity in health care utilization in Brazil, 1998-2008. International Journal for Equity in Health, 11 (33), 1-8. Recuperado de https://equityhealthi.biomedcentral. com/articles/10.1 186/1475-9276-11-33. doi: $\underline{10.1186 / 1475-9276-11-33}$

Marin, M. J. S., Lima, E. F. G., Paviotti, A. B., Matsuyama, D. T., Silva, L. K. D., Gonzalez, C., ... \& llias, M. (2010). Aspectos das fortalezas e fragilidades no uso das metodologias ativas de aprendizagem. Revista Brasileira de Educação Médica, 34(1), 1320. Recuperado de http://www.scielo.br/pdf/ rbem/v34n1/a03v34n1.pdf. doi: 10.1590/s0100$\underline{55022010000100003}$

Mendes, E. V. (2012) O cuidado das condições crônicas na atenção primária à saúde: o imperativo da consolidação da estratégia da saúde da família. Brasília: Organização Pan-Americana da Saúde.

Minayo, M. C. S. (2013). O desafio do conhecimento: pesquisa qualitativa em saúde (13a ed.). São Paulo: Hucitec.

Moreira, M. A. (2011). Aprendizagem significativa: a teoria e textos complementares. São Paulo: Livraria da Física.

Organização Pan Americana da Saúde. (2009). Sistemas de saúde com base na atenção primária: estratégia para o desenvolvimento de equipes de Atenção Primária em Saúde (APS). Washington: Autor. Recuperado de http://www 1.paho.org/hq/dmdocuments/2010/APSEstrategias_desenvolvimento_equipes_APS.pdf
Paim, J., Travassos, C., Almeida, C., Bahia, L., \& Macinko, J. (2011). O sistema de saúde brasileiro: história, avanços e desafios. Lancet, 377(9779), 1778-1797. Recuperado de http://www.thelancet.com/pb/assets/ $\mathrm{raw} /$ Lancet/pdfs/brazil/brazilpor 1.pdf

Higa, E. F. R., Passos, A. H. R., lkuno, M. R. M., Bonifácio, L. A., Mestieri, C. P., \& Ismael, R. K. (2013). Reflexões contemporâneas sobre anamnese na visão do estudante de medicina. Revista Brasileira de Educação Médica, 38(3), 314-322. Recuperado de http://www. scielo.br/pdf/rbem/v38n3/05.pdf. doi: 10.1590/ $\underline{\text { so100-55022014000300005 }}$

Starfield, B. (2002). Atenção primária: equilibrio entre necessidades de saúde, serviços e tecnologia. Brasília: Ministério da Saúde. 